\title{
Re: Whole Exome Sequencing of a Consanguineous Turkish Family Identifies a Mutation in GTF2H3 in Brothers with Spermatogenic Failure
}

\author{
Clavijo RI' ${ }^{1}$, Arora $\mathrm{H}^{2}$, Gibbs $\mathrm{E}^{2}$, Cohen $\mathrm{S}^{2}$, Griswold $\mathrm{A}^{3}$, Bakircioglu $\mathrm{E}^{4}$, Bademci $\mathrm{G}^{3}$, Tekin $\mathrm{M}^{3}$, Ramasamy $\mathrm{R}^{2}$ \\ ${ }^{1}$ University of California School of Medicine, Department of Urology, Davis, Sacramento, California, USA \\ ${ }^{2}$ University of Miami Miller School of Medicine, Department of Urology, Miami, Florida, USA \\ ${ }^{3}$ University of Miami John P. Hussman Institute of Human Genetics, Miami, Florida, USA \\ ${ }^{4}$ Unimed Center, İstanbul, Turkiye
}

Urology 2018. pii: S0090-4295(18)30622-8. doi: 10.1016/j.urology.2018.06.031.

\section{EDITORIAL COMMENT}

In this study, the authors investigated the genetic cause of spermatogenetic failure in a consanguineous Turkish family with four infertile and three fertile brothers by using whole exome sequencing (WES). Two brothers were azoospermic with follicle stimulating hormone, luteinizing hormone and total testosterone levels within the normal range. One of them had a pathology report showing maturation arrest. Both have had microdissection testicular sperm extraction operation without sperm recovery. Spermiogram in other two brothers showed oligoasthenoteratozoospermia (OAT) with a sperm count of 2.2 and 7 million/mL. DNA extraction was obtained for WES in all infertile brothers and parents, who were first-degree cousins, to investigate possible candidate gene(s) which passed to the siblings via Mendelian inheritance. A non-synonymous variant in general transcription factor 2H subunit 3 (GTF2H3) in chromosome 12 was identified in the family. This variant was confirmed to be homozygous in the two azoospermic brothers and heterozygous in the two brothers with OAT. GTF2H3 codes for the protein subunit p34 which comprises part of the GTF2H and this transcription factor has been shown to play a critical role in activating retinoic acid receptor alpha (RAR alpha). RAR alpha has also been shown to be essential in spermatogenesis in human. In this study, the authors proposed that GTF2H3 variant may lead a dysfunction of transcription factor $2 \mathrm{H}$ activation of RAR alpha leading to spermatogenetic failure.

Emre Bakırcıoğlu, MD

๑Copyright 2018 by the Association of Urological Surgery / Journal of Urological Surgery published by Galenos Publishing House. 